\title{
II. BIOAUTOMATICS AND BIOINFORMATICS \\ MATHEMATICAL MODELLING OF A TWO-STAGE ANAEROBIC DIGESTION PROCESS WITH HYDROGEN AND METHANE PRODUCTION USING ADM1
}

\author{
Milen Borisov, Dencho Denchev, Ivan Simeonov
}

\begin{abstract}
The aim of this study is to implement a mathematical model to simulate the dynamic behaviour of a twostage anaerobic digestion process for simultaneous production of hydrogen and methane. The process is carried out in two connected continuously stirred bioreactors. The proposed model is developed by reducing the well known IWA Anaerobic Digester Model No 1 (ADM1). In the present study the original model concept was adapted and applied to replicate a two-stage process. The proposed model involves 13 ODEs for the $1^{\text {st }}$ stage and 7 ODEs for the $2^{\text {nd }}$ stage. The numerical coefficient values in the model are taken from specified literature and adapted to the case of wheat straw AD. Important input-output static characteristics and existence of maxima of the input-output static characteristics concerning the biohydrogen and biomethane production in function of the control variable (dilution rate) are presented. Supposing that both bioreactors are operating nearby these maxima the optimal ratio of the working volumes was obtained. Numerical simulations using a specially elaborated web-based software environment are presented to demonstrate the dynamic behavior of the model solutions.
\end{abstract}

Keywords: anaerobic two-stage digestion; hydrogen and methane production; mathematical model; ADM1, static characteristics, simulation

\section{INTRODUCTION}

Anaerobic digestion (AD) of organic wastes has become a very attractive biotechnology during last years, mainly in the field of the renewable energy sources and biofuels. However, this biotechnology is very useful for depollution of highly polluted with organics wasted waters and municipal wastes. It is known that more than $95 \%$ of working industrial biogas plants operate with the so called continuously stirred tank bioreactors (CSTRs).

$\mathrm{AD}$ is a multi-step biotechnological process with hydrogen $\left(\mathrm{H}_{2}\right)$ as a non-accumulating intermediate product (Ahring, 2003; Deublein, 2008). Recently, the interest in $\mathrm{H}_{2}$ production through $\mathrm{AD}$, also known as dark fermentative $\mathrm{H}_{2}$ production, has increased (Guo et al., 2010; Pakarinen et al., 2011; Ruggeri et al, 2015). This is due to the fact that the rates of $H_{2}$ production are rather high and a variety of feedstock can be used as a substrate. In traditional $\mathrm{AD}, \mathrm{H}_{2}$ is not detected as it is consumed immediately e. g. by hydrogenotrophic methanogens to produce methane $\mathrm{CH}_{4}$ and carbon dioxide $\mathrm{CO}_{2}$ (Gerardi, 2003). On the other hand, $\mathrm{H}_{2}$ can be produced separately by engineering the process conditions. However, the main limitation of dark fermentative $\mathrm{H}_{2}$ production is the rather low energy recovery. In order to completely utilize the organic acids produced during dark fermentation and improve the over-all energy conversion efficiency, a two-stage $\mathrm{AD}$ (TSAD) concept consisting of hydrogenic process followed by methanogenic process has been suggested (Pakarinen et al., 2011).
A lot of models describing separately the fermentative hydrogen production (Nasr et al., 2013; Wang et al., 2009) and the AD for methane production (Batstone et al., 2002; Dochain, 2001; Simeonov, 2010) are known. However, only few models of TSAD processes are known (Simeonov, 2016; Borisov et al., 2016). In (Blumensaat and Keller, 2005) modelling of TSAD using the IWA Anaerobic Digestion Model No 1 (ADM1) is presented. However, the described AD process is not real TSAD process because methane is obtained from both of bioreactors. Volumes of bioreactors are presented different on a figure but without discussing the ratio between them. In experimental studies of co-digestion of mixtures of milk whey and wasted activated sludge in a TSAD process and modelling of this process is presented, however methane is obtained from both of bioreactors [36].

In this paper a new mathematical model describing the process of simultaneous $\mathrm{H}_{2}$ and $\mathrm{CH}_{4}$ production from organic wastes in a cascade of two CSTRs is presented. The proposed model is developed by reducing the universal but very complicated ADM1 basic structure elaborated by the IWA, (Batstone et al., 2002).

The aim of this study is, as well, to investigate the input-output static characteristics and to shows existence of maxima with respect to hydrogen and methane. This fact is important for the practical applications and will be further used in optimising the bioreactors to achieve maximal production of both hydrogen and methane. The model also allows finding 
the optimal ratio between the volumes of the two bioreactors subject to the same optimisation goal.

\section{PROCESS DESCRIBTION}

The application of a TSAD process for simultaneous $\mathrm{H}_{2}$ and $\mathrm{CH}_{4}$ production has been proposed as a promising technology for better process performance and higher energy yields as compared to the traditional one-stage $\mathrm{CH}_{4}$ production process. In the two-stage $\mathrm{AD}$ system, relatively fast growing acidogens and $\mathrm{H}_{2}$ producing microorganisms are developed in the first-stage hydrogenic bioreactor (with working volume $V_{1}$ ) and are involved in the production of volatile fatty acids (VFA) and $H_{2}$. On the other hand, the slow growing acetogens and methanogens are developed in the second-stage methanogenic bioreactor (with working volume $V_{2}$ ) in which the produced VFA are further converted to $\mathrm{CH}_{4}$ and $\mathrm{CO}_{2}$. The scheme of TSAD is shown on Fig. 1.

It is known that in the TSAD $\mathrm{H}_{2}+\mathrm{CH}_{4}$ system the energy yields are up to $43 \%$ more, as compared to the traditional one-stage $\mathrm{CH}_{4}$ production process
(Schievano et al., 2014; Ruggeri, et al, 2015).

In the TSAD system relatively fast growing acidogens and $\mathrm{H}_{2}$ producing microorganisms are grown in the first-stage, the hydrogenic bioreactor (BR1 with working volume $\mathrm{V}_{1}$ ). They are involved in the production of volatile fatty acids (VFAs) and $\mathrm{H}_{2}$. On the other hand, the slow growing acetogens and methanogens are grown in the second stage, the methanogenic bioreactor $\left(\mathrm{BR}_{2}\right.$ with working volume $\mathrm{V}_{2}$ ) in which the produced VFAs are further converted to $\mathrm{CH}_{4}$ and $\mathrm{CO}_{2}$.

The biochemical processes in the first bioreactor (BR1) include (Table 1) disintegration of organic wastes (composites), hydrolysis (of carbohydrates, proteins and lipids), acidogenesis (from sugars and amino acids) and acetogenesis from long chain fatty acids (LCFA) with hydrogen production.

Acetogenesis (from valerate, butyrate and propionate) and the methane production from acetate (methanogenesis) is separated in the second bioreactor (BR2) (Table 2).

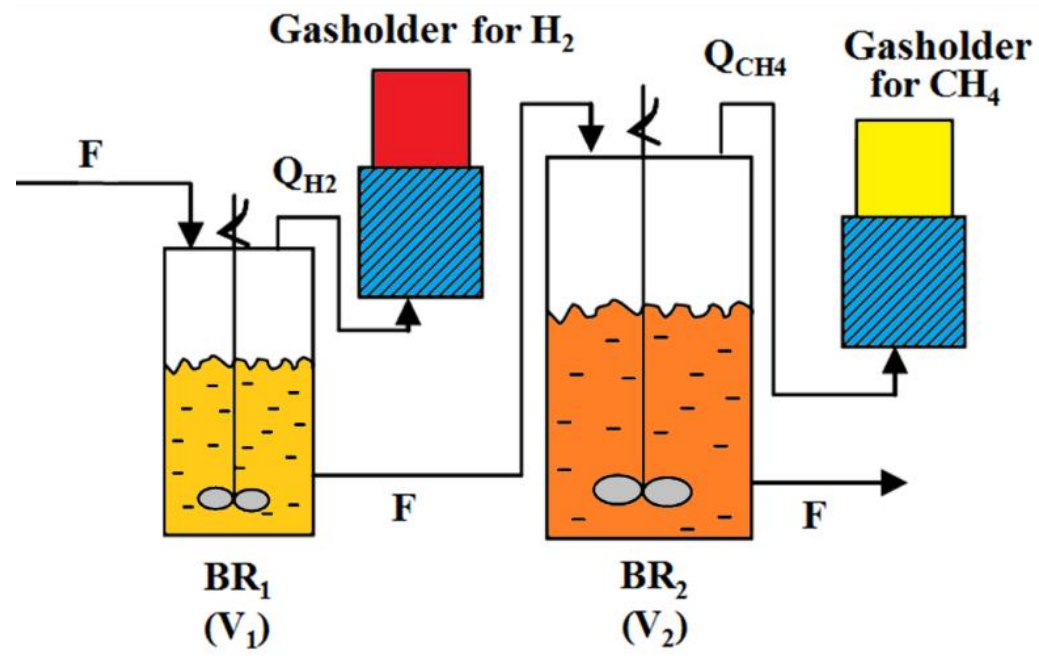

Fig. 1. Scheme of TSAD with production of hydrogen $\left(\mathrm{H}_{2}\right)$ and methane $\left(\mathrm{CH}_{4}\right)$

Assume that the volumes $V_{1}$ and $V_{2}$ of the bioreactors are constant. Let $F_{1}$ and $F_{2}$ be the inflows in the first and second bioreactor respectively and let $F_{1}=F_{2}=F$ be valid. It is well known that the dilution rates $D_{1}$ and $D_{2}$ are defined as:

$$
D_{1}=\frac{F}{V_{1}} \text { and } D_{2}=\frac{F}{V_{2}} \text {. }
$$

After some transformations it was obtained:

$$
\gamma=\frac{V_{1}}{V_{2}}=\frac{D_{2}}{D_{1}} .
$$

It is known that the volume $V_{2}$ of the second bioreactor for methane production is larger than the volume $V_{1}$ of the first bioreactor. Therefore, $\gamma<1$ should be valid. Later on, in the paper we shall determine the constant $\gamma$ using the proposed model equations and one idea for maximal energy production.

Data from experimental studies in laboratory scale of our team are presented [9]. AD of lignocellulosic waste (wheat straw) in single and two-stage processes with production of hydrogen through the acidogenic phase and of methane through the methanogenic phase is studied. As a 
result, the total amount of energy that is produced in the single-stage methanogenic process is $34 \%$ less compared to a two-staged one. This difference comes from both: hydrogen released - about $17 \%$, together with the increased amount of methane about $13 \%$.

Table 1. Biochemical processes in BR1

\begin{tabular}{|c|c|c|}
\hline & Process in BR1 $\left.\mathbf{H}_{\mathbf{2}}\right)$ & Process reaction \\
\hline 1 & Disintegration of composites & $X_{c} \Rightarrow X_{c h}+X_{p r}+X_{l i}$ \\
\hline 2 & Hydrolysis of carbohydrates & $X_{c h} \Rightarrow S_{s u}$ \\
\hline 3 & Hydrolysis of proteins & $X_{p r} \Rightarrow S_{a a}$ \\
\hline 4 & Hydrolysis of lipids & $X_{l i} \Rightarrow S_{s u}+S_{f a}$ \\
\hline 5 & Acidogenesis from sugars & $S_{s u} \stackrel{X_{s u a a}}{\Longrightarrow} S_{a c}+S_{b u}+S_{p r o}+S_{h 2}$ \\
\hline 6 & Acidogenesis from amino acids & $S_{a a} \stackrel{X_{s u a a}}{\longrightarrow} S_{a c}+S_{v a}+S_{b u}+S_{p r o}+S_{h 2}$ \\
\hline 7 & Acetogenesis from LCFA (Long Chan Fatty Acids) & $S_{f a} \stackrel{X_{f a}}{\Longrightarrow} S_{a c}+S_{h 2}$ \\
\hline
\end{tabular}

Table 2. Biochemical processes in BR2

\begin{tabular}{|c|c|c|}
\hline & Process in BR2 $\left(\mathbf{C H}_{4}\right)$ & Process reaction \\
\hline 1 & Acetogenesis from valerate & $S_{v a} \stackrel{X_{c 4}}{\Rightarrow} S_{p r o}+S_{a c}$ \\
\hline 2 & Acetogenesis from butyrate & $S_{b u} \stackrel{X_{c 4}}{\Rightarrow} S_{a c}$ \\
\hline 3 & Acetogenesis from propionate & $S_{p r o} \stackrel{X_{p r o}}{\Longrightarrow} S_{a c}$ \\
\hline 4 & Methanogenesis form acetate & $S_{a c} \stackrel{X_{a c}}{\Rightarrow} S_{c h 4}$ \\
\hline
\end{tabular}

\section{PROCESS MODEL}

The model is derived on the basis of the ADM1 basic structure as well as on our experience with TSAD process with hydrogen and methane production (Denchev et al., 2016). The following assumptions have been accepted:

- Balance equations of the hydrogen and methane in the liquid phases have been neglected because they are practically not dissolved in liquids.

- Hydrogenotrophic bacteria do not exist in this process.

- Equations describing balances of inorganic components and some biochemical equations have been neglected in view of simplifying the model.

- The very important parameter $p H$ is not included in the model, however $p H$ is kept in the interval $5.0-5.5$ in the first bioreactor, and in the interval $6.5-8.5$ in the second bioreactor.

- The biochemical processes in BR1 include disintegration of organic wastes (composites), hydrolysis (of carbohydrates, proteins and lipids), acidogenesis (from sugars and amino acids) and acetogenesis from LCFA with hydrogen production. These processes are omitted in BR2.

- Acetogenesis (from valerate, butyrate and propionate) and the methane production from acetate (methanogenesis) are separated in the BR2.

Following the above presented assumptions, the dynamics in the BR1 is described by the following set of 13 nonlinear ordinary differential equations (ODEs):

$$
\begin{aligned}
& \frac{d}{d t} S_{s u, R H_{2}}(t)=D_{R H_{2}}\left(S_{s u, R H_{2}, i n}-S_{s u, R H_{2}}\right)+k_{h y d, c h} X_{c h, R H_{2}}+f_{s u, l i} k_{h u d, l i} X_{l i, R H_{2}} \\
& -k_{m, \text { suaa }} \frac{S_{s u, R H_{2}} X_{\text {suaa }, R H_{2}}}{K_{S, \text { suaa }}+S_{s u, R H_{2}}} \frac{S_{s u, R H_{2}}}{S_{s u, R H_{2}}+S_{a a, R H_{2}}} \\
& \frac{d}{d t} S_{a a, R H_{2}}(t)=D_{R H_{2}}\left(S_{a a, R H_{2}, i n}-S_{a a, R H_{2}}\right)+k_{h y d, p r} X_{p r, R H_{2}}-\frac{S_{a a, R H_{2}} X_{s u a a, R H_{2}}}{K_{S, s u a a}+S_{a a, R H_{2}}} \frac{k_{m, s u a a} S_{a a, R H_{2}}}{S_{s u, R H_{2}}+S_{a a, R H_{2}}} \\
& \frac{d}{d t} S_{f a, R H_{2}}(t)=D_{R H_{2}}\left(S_{f a, R H_{2}, i n}-S_{f a, R H_{2}}\right)+f_{f a, l i} k_{h u d, l i} X_{l i, R H_{2}}-k_{m, f a} \frac{S_{f a, R H_{2}} X_{f a, R H_{2}}}{K_{S, f a}+S_{f a, R H_{2}}}
\end{aligned}
$$




$$
\begin{aligned}
& \frac{d}{d t} S_{v a, R H_{2}}(t)=D_{R H_{2}}\left(S_{v a, R H_{2}, \text { in }}-S_{v a, R H_{2}}\right)+\left(1-Y_{\text {suaa }}\right) f_{v a, a a} k_{m, \text { suaa }} \frac{S_{a a, R H_{2}} X_{s u a a, R H_{2}}}{K_{S, \text { suaa }}+S_{a a, R H_{2}}} \\
& \frac{d}{d t} S_{b u, R H_{2}}(t)=D_{R H_{2}}\left(S_{b u, R H_{2}, \text { in }}-S_{b u, R H_{2}}\right) \\
& +\left(1-Y_{\text {suaa }}\right) k_{m, \text { suaa }}\left(\frac{S_{s u, R H_{2}} X_{s u a a, R H_{2}}}{K_{S, \text { suaa }}+S_{s u, R H_{2}}} \frac{f_{b u, s u} S_{s u, R H_{2}}}{S_{s u, R H_{2}}+S_{a a, R H_{2}}}+\frac{S_{a a, R H_{2}} X_{s u a a, R H_{2}}}{K_{S, s u a a}+S_{a a, R H_{2}}} \frac{f_{b u, a a} S_{a a, R H_{2}}}{S_{s u, R H_{2}}+S_{a a, R H_{2}}}\right) \\
& \frac{d}{d t} S_{\text {pro, } R H_{2}}(t)=D_{R H_{2}}\left(S_{\text {pro, } R H_{2}, \text { in }}-S_{\text {pro }, R H_{2}}\right)
\end{aligned}
$$

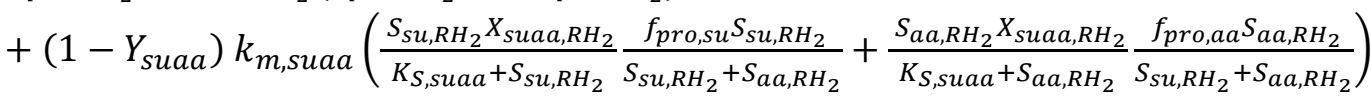

$$
\begin{aligned}
& \frac{d}{d t} S_{a c, R H_{2}}(t)=D_{R H_{2}}\left(S_{a c, R H_{2}, i n}-S_{a c, R H_{2}}\right)+\left(1-Y_{f a}\right) 0.7 k_{m, f a} \frac{S_{f a, R H_{2}}}{K_{S, f a}+S_{f a_{1} R H_{2}}} X_{f a, R H_{2}} v \\
& +\left(1-Y_{\text {suaa }}\right) k_{m, \text { suaa }}\left(\frac{S_{s u, R H_{2}} X_{s u a a, R H_{2}}}{K_{S, \text { sua }}+S_{s u, R H_{2}}} \frac{f_{a c, s u} S_{s u, R H_{2}}}{S_{s u, R H_{2}}+S_{a a, R H_{2}}}+\frac{S_{a a, R H_{2}} X_{s u a a, R H_{2}}}{K_{S, \text { suaa }}+S_{a a, R H_{2}}} \frac{f_{a c, a a} S_{a a, R H_{2}}}{S_{s u, R H_{2}}+S_{a a, R H_{2}}}\right) \\
& \frac{d}{d t} X_{C, R H_{2}}(t)=D_{R H_{2}}\left(X_{C, R H_{2}, \text { in }}-X_{c, R H_{2}}\right)-k_{d i s} X_{c, R H_{2}} \\
& \frac{d}{d t} X_{c h, R H_{2}}(t)=D_{R H_{2}}\left(X_{c h, R H_{2}, i n}-X_{c h, R H_{2}}\right)+f_{c h, x c} k_{d i s} X_{c, R H_{2}}-k_{h y d, c h} X_{c h, R H_{2}} \\
& \frac{d}{d t} X_{p r, R H_{2}}(t)=D_{R H_{2}}\left(X_{p r, R H_{2}, i n}-X_{p r, R H_{2}}\right)+f_{p r, x c} k_{d i s} X_{c, R H_{2}}-k_{h y d, p r} X_{p r, R H_{2}} \\
& \frac{d}{d t} X_{l i, R H_{2}}(t)=D_{R H_{2}}\left(X_{l i, R H_{2}, i n}-X_{l i, R H_{2}}\right)+f_{l i, x c} k_{d i s} X_{c, R H_{2}}-k_{h y d, l i} X_{l i, R H_{2}} \\
& \frac{d}{d t} X_{\text {suaa }, R H_{2}}(t)=D_{R_{2}}\left(X_{\text {suaa }, R H_{2}, \text { in }}-X_{\text {suaa }, R H_{2}}\right)
\end{aligned}
$$

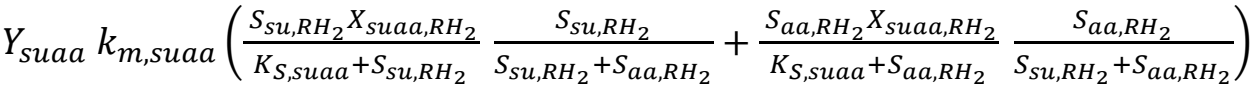

$$
\begin{aligned}
& \frac{d}{d t} X_{f a, R H_{2}}(t)=D_{R H_{2}}\left(X_{f a, R H_{2}, i n}-X_{f a, R H_{2}}\right)+Y_{f a} k_{m, f a} \frac{S_{f a, R H_{2}} X_{f a, R H_{2}}}{K_{S, f a}+S_{f a, R H_{2}}} \\
& Q_{h 2}(t)=Y_{h 2, s u} k_{m, \text { suaa }} \frac{S_{s u, R H_{2}} X_{\text {suaa }, R H_{2}}}{K_{S, \text { suaa }}+S_{\text {su }, R H_{2}}} \frac{S_{s u, R H_{2}}}{S_{s u, R H_{2}}+S_{a a, R H_{2}}} \\
& +Y_{h 2, a a} k_{m, \text { suaa }} \frac{S_{a a, R H_{2}} X_{\text {suaa }, R H_{2}}}{K_{S, \text { suaa }}+S_{a a, R H_{2}}} \frac{S_{a a, R H_{2}}}{S_{s u, R H_{2}}+S_{a a, R H_{2}}}+Y_{h 2, f a} k_{m, f a} \frac{S_{f a, R H_{2} X_{f a, R H_{2}}}}{K_{S, f a}+S_{f a, R H_{2}}}
\end{aligned}
$$

The algebraic equation (16) describes the hydrogen formation in the gaseous phase of BR1.

The dynamics of the process in BR2 is described by the following set of 7 ODEs:

$$
\begin{aligned}
& \frac{d}{d t} S_{v a, R C H_{4}}(t)=D_{R C H_{4}}\left(S_{v a, R C H_{4}, i n}-S_{v a, R C H_{4}}\right)-k_{m, C 4} \frac{S_{v a, R C H_{4}} X_{C 4, R C H_{4}}}{K_{S, c 4}+S_{v a, R C H_{4}}} \frac{S_{v a, R C H_{4}}}{S_{b u, R C H_{4}}+S_{v a, R C H_{4}}} \\
& \frac{d}{d t} S_{b u, R C H_{4}}(t)=D_{R C H_{4}}\left(S_{b u, R C H_{4}, i n}-S_{b u, R C H_{4}}\right)-k_{m, c 4} \frac{S_{b u, R C H_{4}} X_{C 4, R C H_{4}}}{K_{S, c 4}+S_{b u, R C H_{4}}} \frac{S_{b u, R C H_{4}}}{S_{b u, R C H_{4}}+S_{v a, R C H_{4}}} \\
& \frac{d}{d t} S_{{\text {pro }, R C H_{4}}_{4}}(t)=D_{R^{2} H_{4}}\left(S_{\text {pro }, R C H_{4}, \text { in }}-S_{\text {pro }, R_{C H}}\right)
\end{aligned}
$$

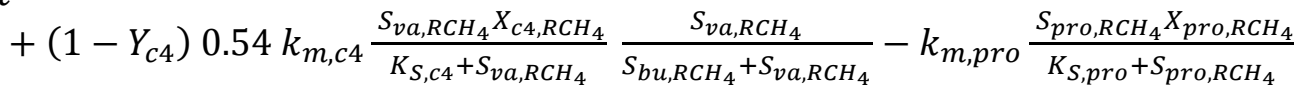

$$
\begin{aligned}
& \frac{d}{d t} S_{a c, R C H_{4}}(t)=D_{R C H_{4}}\left(S_{a c, R C H_{4}, \text { in }}-S_{a c, R C H_{4}}\right) \\
& +\left(1-Y_{c 4}\right) k_{m, c 4}\left(0.31 \frac{S_{v a, R C H_{4}} X_{C 4, R C H_{4}}}{K_{S, C 4}+S_{v a, R C H_{4}}} \frac{S_{v a, R C H_{4}}}{S_{b u, R C H_{4}}+S_{v a, R C H_{4}}}+0.8 \frac{S_{b u, R C H_{4}} X_{C 4, R C H_{4}}}{K_{S, C 4}+S_{b u, R C H_{4}}} \frac{S_{b u, R C H_{4}}}{S_{b u, R C H_{4}}+S_{v a, R C H_{4}}}\right) \\
& +\left(1-Y_{\text {pro }}\right) 0.57 k_{m, \text { pro }} \frac{S_{p r o, R C H_{4}} X_{\text {pro }, R C H_{4}}}{K_{S, p r o}+S_{p_{\text {ro }} \mathrm{RCH}_{4}}}-Y_{a c} k_{m, a c} \frac{S_{a c, R C H_{4}} X_{a c, R C H_{4}}}{K_{S, a c}+S_{a c, R C H_{4}}}
\end{aligned}
$$


$\frac{d}{d t} X_{C 4, R C H_{4}}(t)=D_{R C H_{4}}\left(0-X_{C 4, R C H_{4}}\right)+Y_{c 4} k_{m, c 4} \frac{S_{v a, R C H_{4}} X_{C 4, R C H_{4}}}{K_{S, C 4}+S_{v a, R C H_{4}}} \frac{s_{v a, R C H_{4}}}{S_{b u, R C H_{4}}+S_{v a, R C H_{4}}}$

$+Y_{C 4} k_{m, C 4} \frac{S_{b u, R C H} X_{C 4, R C H}}{K_{S, C 4}+S_{b u, R C H}} \frac{S_{b u, R C H}}{S_{b u, R C H}+S_{v a, R C H}}$

$\frac{d}{d t} X_{\text {pro }, \mathrm{RCH}_{4}}(t)=D_{\mathrm{RCH}_{4}}\left(0-X_{\text {pro }, \mathrm{RCH}_{4}}\right)+Y_{\text {pro }} k_{m, \text { pro }} \frac{S_{\text {pro }, \mathrm{RCH}} \mathrm{X}_{\text {pro }, \mathrm{RCH} \mathrm{H}_{4}}}{K_{S, p r o}+S_{\text {pro }, \mathrm{RCH}}}$

$\frac{d}{d t} X_{a c, R C H_{4}}(t)=D_{R C H_{4}}\left(0-X_{a c, R C H_{4}}\right)+k_{m, a c} \frac{S_{a c, R C H_{4}} X_{a c, R C H_{4}}}{K_{S, a c}+S_{a c, R C H_{4}}}$

$Q_{c h 4}(t)=Y_{c h 4, a c} k_{m, a c} \frac{S_{a c, R C H_{4}} X_{a c, R C H_{4}}}{K_{S, a c}+S_{a c, R C H_{4}}}$

The algebraic equation (24) describes the methane formation in the gaseous phase of BR2, where:

$S_{v a, R C H_{4}, \text { in }}=S_{v a, R H_{2}}, S_{b u, R C H_{4}, \text { in }}=S_{b u, R H_{2}}$,

$S_{\text {pro }, \mathrm{RCH}_{4}, \text { in }}=S_{\text {pro, } \mathrm{RH}}$.

The definitions of the phase variables and

Table 3. Phase variables

\begin{tabular}{|c|c|c|c|}
\hline Eq. & Variable & Component & Processes \\
\hline$(1)$ & $S_{s u, R H_{2}}$ & Monosaccharides & + hydrolysis of carbohydrates, lipids - uptake of sugar \\
\hline$(2)$ & $S_{a a, R H_{2}}$ & Amino acids (AA) & + hydrolysis of proteins - uptake of amino acid \\
\hline$(3)$ & $S_{f a, R H_{2}}$ & Fatty acids (LCFA) & + hydrolysis of lipids - uptake of LCFA \\
\hline$(4)$ & $S_{v a, R H_{2}}$ & Total valerate & + uptake of amino acids \\
\hline$(5)$ & $S_{b u, R H_{2}}$ & Total butyrate & + uptake of sugar, amino acids \\
\hline$(6)$ & $S_{p r o, R H_{2}}$ & Total propionate & + uptake of sugar, amino acids \\
\hline$(7)$ & $S_{a c, R H_{2}}$ & Total acetate & + uptake of sugar, amino acids, LCFA \\
\hline$(8)$ & $X_{c, R H_{2}}$ & Composites & - disintegration of composites \\
\hline$(9)$ & $X_{c h, R H_{2}}$ & Carbohydrates & + disintegration of composites - hydrolysis of carbohydrates \\
\hline$(10)$ & $X_{p r, R H_{2}}$ & Proteins & + disintegration of composites - hydrolysis of proteins \\
\hline$(11)$ & $X_{l i, R H_{2}}$ & Lipids & + disintegration of composites - hydrolysis of lipids \\
\hline$(12)$ & $X_{s u a a, R H_{2}}$ & Sugar and AA degraders & + uptake of sugar, amino acids) \\
\hline$(13)$ & $X_{f a, R H_{2}}$ & LCFA degraders & + uptake of LFCA \\
\hline$(14)$ & $Q_{h 2}$ & Hydrogen gas & stripping of hydrogen \\
\hline$(15)$ & $S_{v a, R C H_{4}}$ & Total valerate (Va) & - uptake of valerate \\
\hline$(16)$ & $S_{b u, R C H_{4}}$ & Total butyrate (Bu) & - uptake of butyrate \\
\hline$(17)$ & $S_{p r o, R C H_{4}}$ & Total propionate & + uptake of acetate \\
\hline$(18)$ & $S_{a c, R C H_{4}}$ & Total acetate & stripping of methane \\
\hline$(19)$ & $X_{c 4, R C H_{4}}$ & Va and Bu degraders & + uptake of valerate, butyrate, propionate - acetate \\
\hline$(20)$ & $X_{p r o, R C H_{4}}$ & Propionate degraders & + uptake of valerate, butyrate \\
\hline$(21)$ & $X_{a c, R C H_{4}}$ & Acetate degraders & + uptake of propionate \\
\hline$(22)$ & $Q_{c h 4}$ & Methane gas & \\
\hline
\end{tabular}

parameters in the equations (3)-(24) are given in Table 3 and Table 4, respectively. The coefficient values in the right column of Table 4 are taken from (Rosen et al., 2006). In the model variables and parameters, the subscripts $h 2$ and $c h 4$ indicate hydrogen $\left(\mathrm{H}_{2}\right)$ and methane $\left(\mathrm{CH}_{4}\right)$ respectively. 
Table 4. Parameters values

\begin{tabular}{|c|c|c|}
\hline Parameter & Definition of the model parameters & Values \\
\hline$S_{S u, R H_{2}, \text { in }}$ & input concentration of $S_{\mathrm{Su} \mathrm{RH}}[\mathrm{gCOC} / \mathrm{L}]$ & 0.01 \\
\hline $\mathrm{S}_{a a, \mathrm{RH}_{2}, \mathrm{in}}$ & input concentration of $S_{a a, R H_{2}}[\mathrm{gCOC} / \mathrm{L}]$ & 0.001 \\
\hline $\mathrm{S}_{\mathrm{fa}, \mathrm{RH}, \mathrm{in}}$ & input concentration of $S_{f a, \mathrm{RH}_{2}}[\mathrm{gCOC} / \mathrm{L}]$ & 0.001 \\
\hline$S_{v a, R H_{2}, \text { in }}$ & input concentration of $S_{v a, R H_{2}}[\mathrm{gCOC} / \mathrm{L}]$ & 0.0 \\
\hline$S_{b u, R H_{2}, \text { in }}$ & input concentration of $S_{b u, R H_{2}}[\mathrm{gCOC} / \mathrm{L}]$ & 0.0 \\
\hline$S_{\text {pro, } R H_{2}, \text { in }}$ & input concentration of $S_{p r o, R H_{2}}[\mathrm{gCOC} / \mathrm{L}]$ & 0.0 \\
\hline$S_{a c, R H_{2}, \text { in }}$ & input concentration of $S_{a c, R H_{2}}[\mathrm{gCOC} / \mathrm{L}]$ & 0.0 \\
\hline$X_{C, R H_{2}, \text { in }}$ & input concentration of $X_{C, R H_{2}}[\mathrm{gCOC} / \mathrm{L}]$ & 50.0 \\
\hline $\mathrm{X}_{\mathrm{Ch}, \mathrm{RH}, \mathrm{in}}$ & input concentration of $X_{c h, R H_{2}}[\mathrm{gCOC} / \mathrm{L}]$ & 0.0 \\
\hline$X_{p r, R H_{2}, \text { in }}$ & input concentration of $X_{p r, R H_{2}}[\mathrm{gCOC} / \mathrm{L}]$ & 0.0 \\
\hline$X_{l i, R H_{2}, \text { in }}$ & input concentration of $X_{l i, R H_{2}}[\mathrm{gCOC} / \mathrm{L}]$ & 0.0 \\
\hline $\mathrm{X}_{\text {Suaa }, \mathrm{RH}_{2}, \mathrm{in}}$ & input concentration of $X_{{\text {suaa }, R H_{2}}}[\mathrm{gCOC} / \mathrm{L}]$ & 0.0 \\
\hline$X_{f a, R H_{2}, i n}$ & input concentration of $X_{f a, R H_{2}}[\mathrm{gCOC} / \mathrm{L}]$ & 0.0 \\
\hline$f_{c h, x c}$ & stoichiometric parameter $[-]$ & 0.2 \\
\hline$f_{p r, x c}$ & stoichiometric parameter $[-]$ & 0.2 \\
\hline$f_{l i, x c}$ & stoichiometric parameter $[-]$ & 0.3 \\
\hline$f_{s u, l i}$ & stoichiometric parameter $[-]$ & 0.05 \\
\hline$f_{f a, l i}$ & stoichiometric parameter [-] & 0.95 \\
\hline$f_{a c, s u}$ & stoichiometric parameter [-] & 0.41 \\
\hline$f_{a c, a a}$ & stoichiometric parameter $[-]$ & 0.4 \\
\hline$f_{b u, s u}$ & stoichiometric parameter [-] & 0.13 \\
\hline$f_{\text {pro,su }}$ & stoichiometric parameter [-] & 0.27 \\
\hline$f_{v a, a a}$ & stoichiometric parameter $[-]$ & 0.23 \\
\hline$f_{b u, a a}$ & stoichiometric parameter $[-]$ & 0.26 \\
\hline$f_{\text {pro,aa }}$ & stoichiometric parameter [-] & 0.05 \\
\hline$Y_{\text {suaa }}$ & stoichiometric parameter $[-]$ & 0.1 \\
\hline$Y_{a c}$ & stoichiometric parameter $[-]$ & 27.3 \\
\hline$Y_{f a}$ & stoichiometric parameter $[-]$ & 0.06 \\
\hline$Y_{h 2, s u}$ & physicochemical parameter $\left[\mathrm{L}^{2} / \mathrm{g}\right]$ & 0.7 \\
\hline$Y_{h 2, a a}$ & physicochemical parameter $\left[\mathrm{L}^{2} / \mathrm{g}\right]$ & 0.7 \\
\hline$Y_{h 2, f a}$ & physicochemical parameter $\left[\mathrm{L}^{2} / \mathrm{g}\right]$ & 0.7 \\
\hline$Y_{c h 4, a c}$ & physicochemical parameter $\left[\mathrm{L}^{2} / \mathrm{g}\right]$ & 75 \\
\hline$k_{\text {dis }}$ & biochemical parameter $\left[\mathrm{h}^{-1}\right]$ & 0.0208 \\
\hline$k_{h y d, c h}$ & biochemical parameter $\left[\mathrm{h}^{-1}\right]$ & 0.417 \\
\hline$k_{\text {hyd,pr }}$ & biochemical parameter $\left[\mathrm{h}^{-1}\right]$ & 0.417 \\
\hline$k_{h y d, l i}$ & biochemical parameter $\left[\mathrm{h}^{-1}\right]$ & 0.417 \\
\hline$k_{m, \text { suaa }}$ & biochemical parameter $\left[\mathrm{h}^{-1}\right]$ & 1.25 \\
\hline$k_{s, \text { suaa }}$ & biochemical parameter $\left[\mathrm{h}^{-1}\right]$ & 0.5 \\
\hline$k_{m, f a}$ & biochemical parameter $\left[\mathrm{h}^{-1}\right]$ & 0.15 \\
\hline$k_{m, c a}$ & biochemical parameter $\left[\mathrm{h}^{-1}\right]$ & 0.0167 \\
\hline$k_{S, f a}$ & biochemical parameter $\left[\mathrm{h}^{-1}\right]$ & 0.67 \\
\hline$k_{S, a c}$ & biochemical parameter $\left[\mathrm{h}^{-1}\right]$ & 0.4 \\
\hline$k_{m, c 4}$ & biochemical parameter $\left[\mathrm{h}^{-1}\right]$ & 0.833 \\
\hline$K_{S, c 4}$ & biochemical parameter $[\mathrm{g} / \mathrm{L}]$ & 0.2 \\
\hline$k_{m, \text { pro }}$ & biochemical parameter $\left[\mathrm{h}^{-1}\right]$ & 0.542 \\
\hline$K_{S, p r o}$ & biochemical parameter [g/L] & 0.1 \\
\hline
\end{tabular}




\section{INPUT OUTPUT STATIC CHARACTERISTICS}

The function $Q_{h 2}\left(D_{1}\right)$ depends on the input $D_{1}$ and is called input-output static characteristic with respect to the hydrogen production. Fig. 2 presents the graph of the input-output static characteristic for three value of the parameter $X_{c}^{(i n)}=X_{c, R H_{2}, \text { in }}$. From this figure is evident that $Q_{h 2}\left(D_{1}\right)$ is unimodal with respect to $D_{1}$ in the admissible interval for $D_{1}$ and there exist a unique (admissible) point $D_{1, \max }$, such that $Q_{h 2}\left(D_{1}\right)$ possesses maximum $Q_{h 2, \max }\left(Q_{h 2, \max }=\right.$ $\left.Q_{h 2}\left(D_{1, \max }\right)\right), Q_{h 2}\left(D_{1}\right)$ is strongly increasing if $D_{1} \leq$ $D_{1, \text { max }}$ and $Q_{h 2}\left(D_{1}\right)$ is strongly decreasing if $D_{1}>D_{1, \text { max }}$.

\section{SIMULATION STUDIES}

Fig. 4 and Fig. 5 present the time evolution of the outputs $Q_{h 2}(t)$ and $Q_{c h 4}(t)$, respectively, $X_{c}^{(i n)}$ takes values from Table 6 for $D_{1}=0.025=$ const.
Using the methane flow rate $Q_{c h 4}$ we compute the input-output static characteristic on the set of all steady states, namely: $Q_{c h 4}=Q_{c h 4}\left(D_{1}, D_{2}\right)$.

Fig. 3 visualizes the graph of $Q_{c h 4}\left(D_{1, \max }, D_{2}\right)$; the latter is a unimodal function, taking its maximum at $D_{2, \max } ; Q_{c h 4, \max }=Q_{\mathrm{ch} 4}\left(D_{1, \max }, D_{2, \max }\right)$.

Using the presentation (2) we define and compute the constant $\gamma$ and in this way the relationship between the volumes $V_{1}$ and $V_{2}$ of the two bioreactors (Table 5).

For simulations the average value (from the last column) $\gamma=0.253$ was adopted (the volume of BR2 is about 4 times bigger than those of BR1).

Table 5. Values for the constant $\gamma$ for different values of $X \_c^{\wedge}($ (in))

\begin{tabular}{|c|c|c|c|c|c|c|c|}
\hline $\mathbf{X}_{\mathbf{c}}^{(\mathbf{i n )}}[\mathrm{g} / \mathrm{L}]$ & $\mathbf{D}_{\mathbf{1 , \text { sup }}}[1 / \mathrm{h}]$ & $\mathbf{D}_{\mathbf{1 , \operatorname { m a x }}}[1 / \mathrm{h}]$ & $\mathbf{Q}_{\mathbf{1 , \operatorname { m a x }}}[\mathrm{L} / \mathrm{h}]$ & $\mathbf{D}_{\text {2,sup }}[1 / \mathrm{h}]$ & $\mathbf{D}_{\text {2,max }}[1 / \mathrm{h}]$ & $\mathbf{Q}_{\mathbf{2 , \text {max}}}[\mathrm{L} / \mathrm{h}]$ & $\boldsymbol{\gamma}$ \\
\hline 40 & 0.089 & 0.041 & 0.132 & 0.0145 & 0.0106 & 0.0608 & 0.258 \\
\hline 50 & 0.093 & 0.044 & 0.169 & 0.0150 & 0.0111 & 0.0774 & 0.252 \\
\hline 60 & 0.097 & 0.046 & 0.206 & 0.0153 & 0.0115 & 0.0953 & 0.249 \\
\hline
\end{tabular}

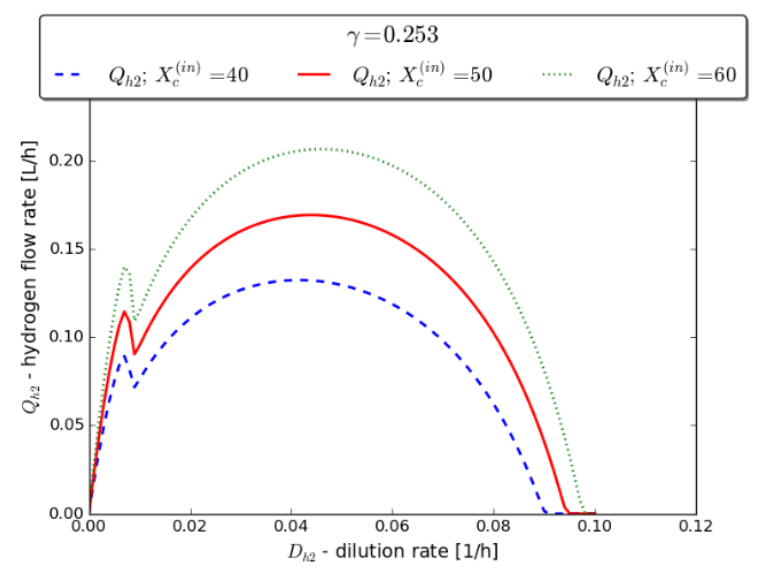

Fig. 2. Input-output static characteristics for BR1

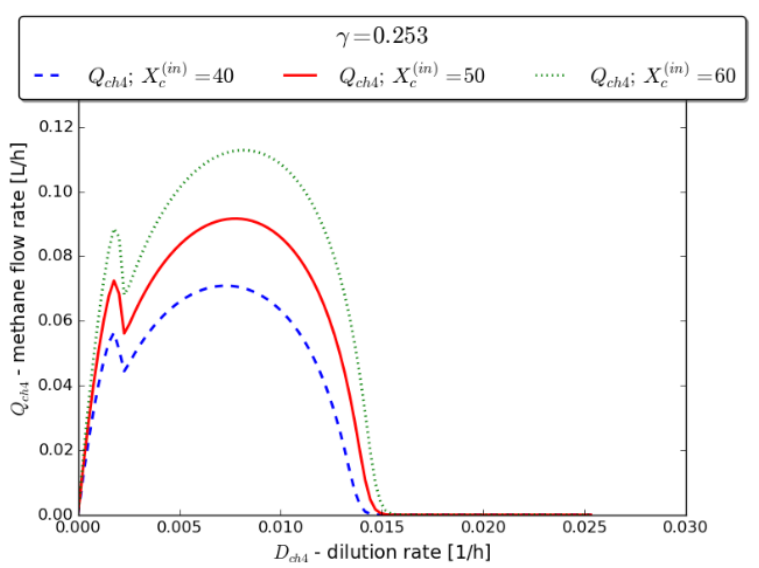

Fig. 3. Input-output static characteristics for BR2

Table 6. Values of $X_{c}^{(i n)}$ for $D_{1}=0.025=$ const., $D_{2}=\gamma D_{1}$, where $\gamma=0.253$

\begin{tabular}{|c|c|c|c|c|c|}
\hline Time & Hour $[\mathbf{h}]$ & $\mathbf{0 - 1 0 0 0}$ & $\mathbf{1 0 0 0 - 2 0 0 0}$ & $\mathbf{2 0 0 0 - 3 0 0 0}$ & $\mathbf{3 0 0 0}-\mathbf{4 0 0 0}$ \\
\hline$X_{c}^{(i n)}$ & {$[\mathrm{g} / l]$} & 50 & 40 & 60 & 50 \\
\hline
\end{tabular}




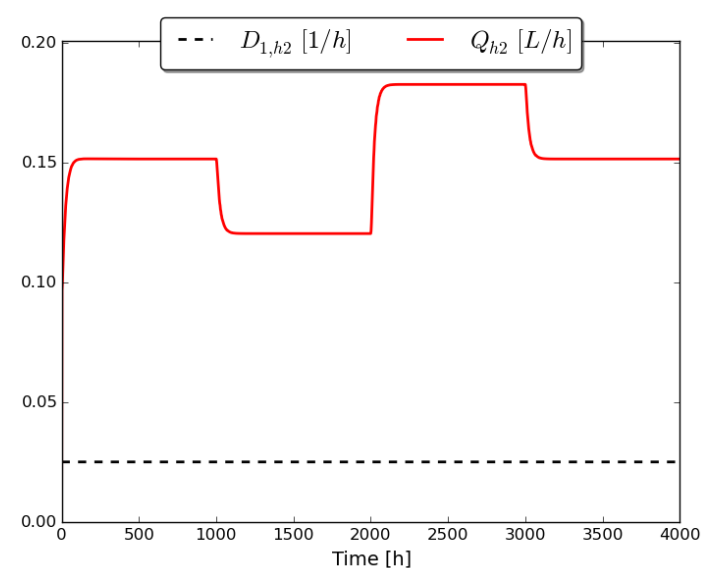

Fig. 4

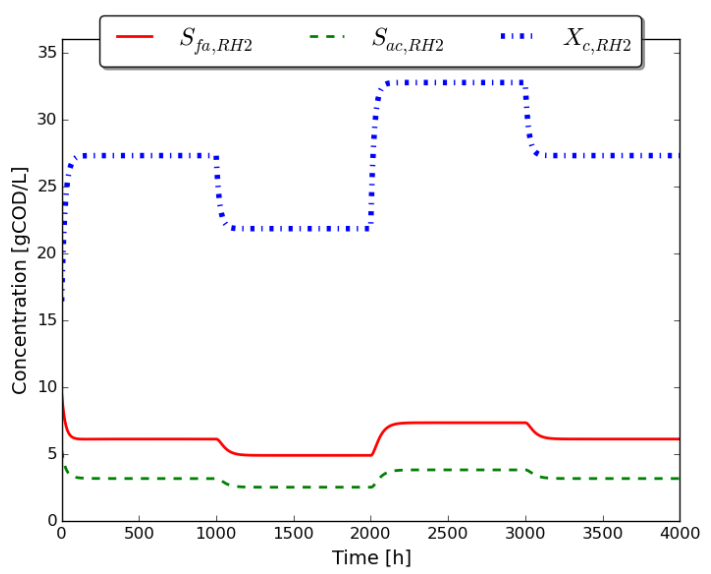

Fig. 6

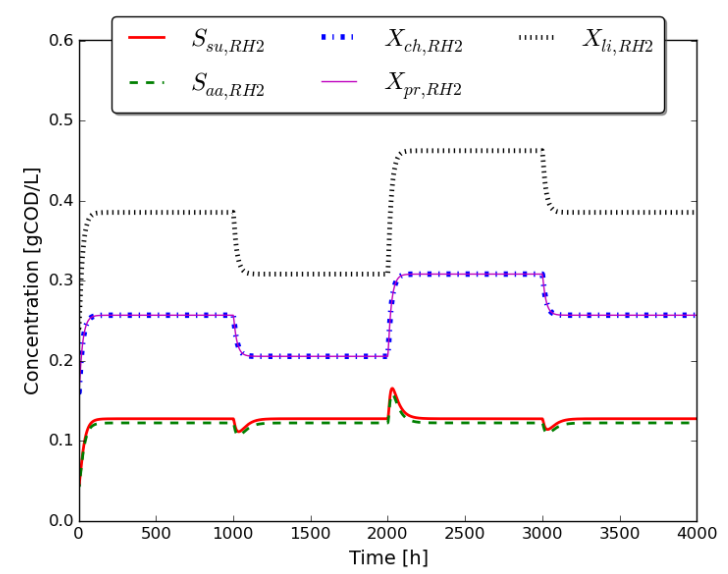

Fig. 8

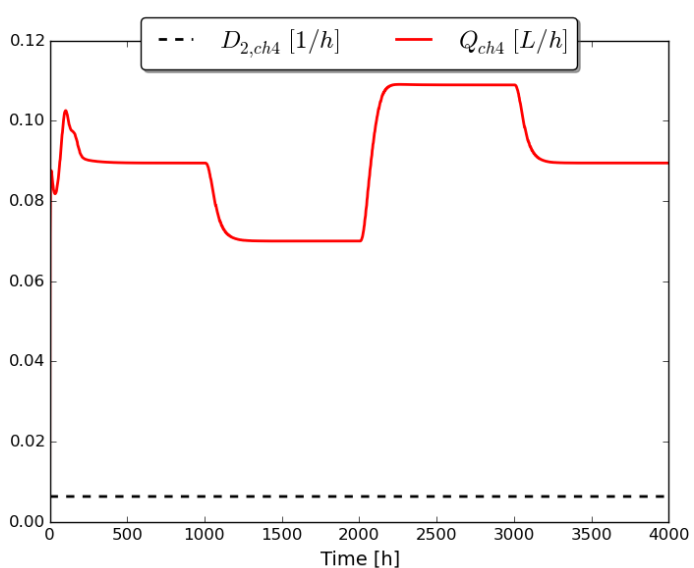

Fig. 5

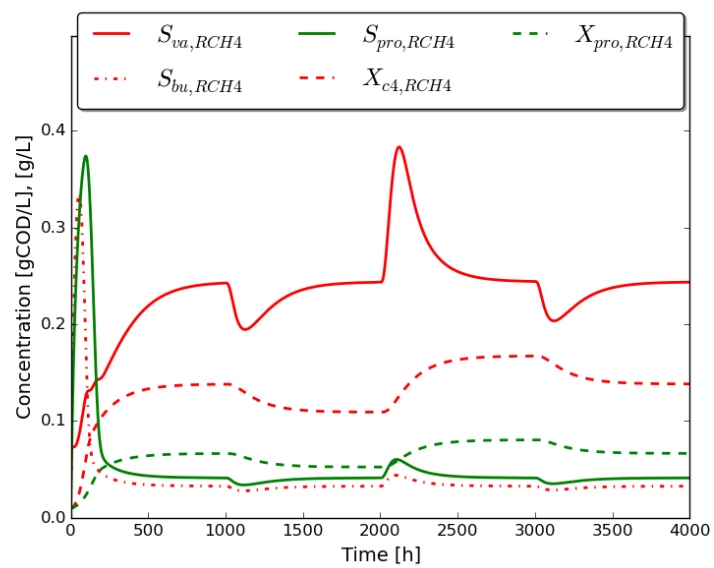

Fig. 7

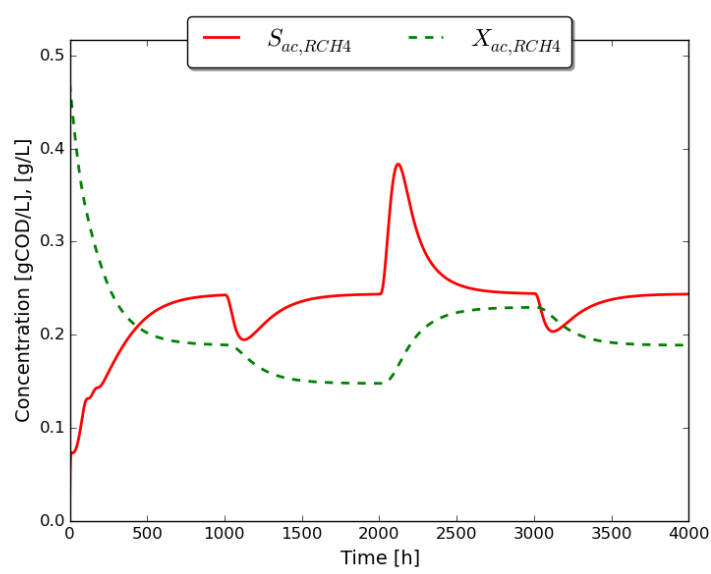

Fig. 9 


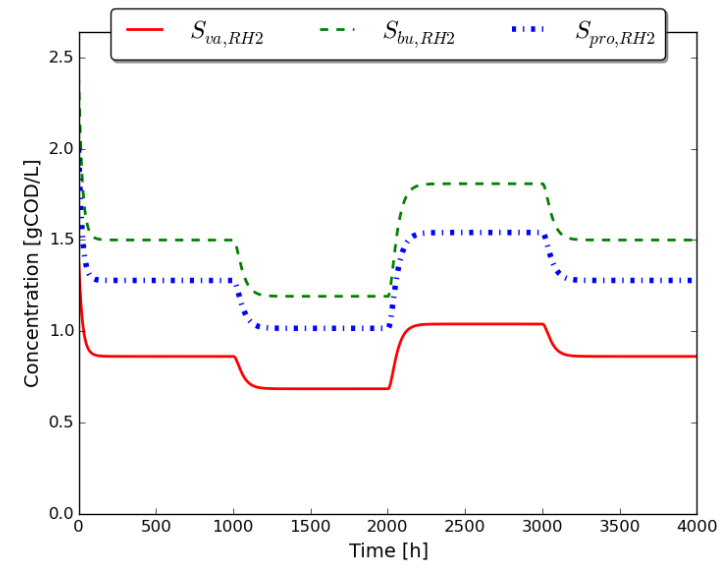

Fig. 10

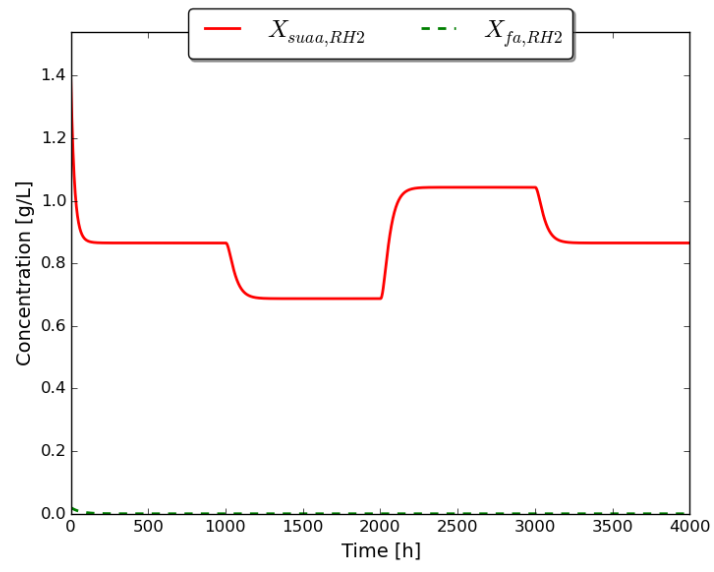

Fig. 11

Fig. 12 to Fig. 19 present the time evolution of the model solutions, where $D_{1}$ takes values from

Table 7 for $X_{c}^{(i n)}=50=$ const., $\gamma=0.253$.

Table 7. Step changes of $D_{1}$ with $D_{2}=\gamma D_{1}, X_{c}^{(i n)}=50=$ const. $(\gamma=0.253, K=1 / \gamma \approx 3.95)$

\begin{tabular}{|c|c|c|c|c|c|c|c|}
\hline Time & Hour $[\mathbf{h}]$ & $\mathbf{0}-\mathbf{1 0 0 0}$ & $\mathbf{1 0 0 0 - \mathbf { 2 0 0 0 }}$ & $\mathbf{2 0 0 0 - \mathbf { 3 0 0 0 }}$ & $\mathbf{3 0 0 0}-\mathbf{4 0 0 0}$ & $\mathbf{4 0 0 0 - \mathbf { 5 0 0 0 }}$ & $\mathbf{5 0 0 0}-\mathbf{8 0 0 0}$ \\
\hline$D_{1}$ & {$[1 / h]$} & 0.01 & 0.015 & 0.02 & 0.025 & 0.035 & 0.1 \\
\hline
\end{tabular}

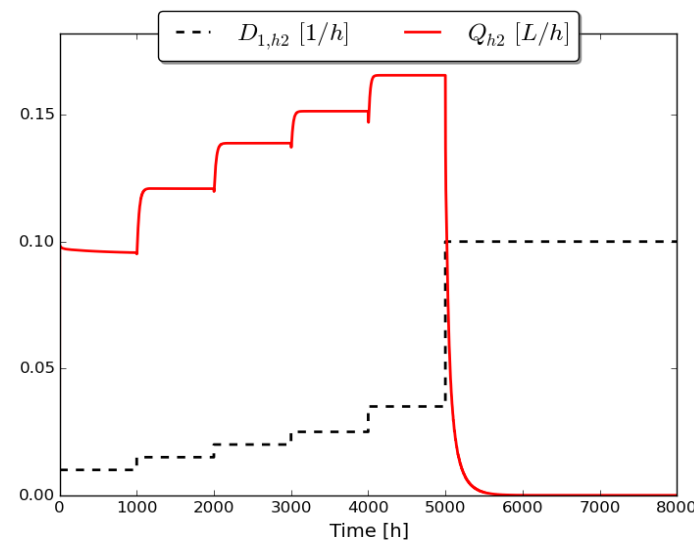

Fig. 12

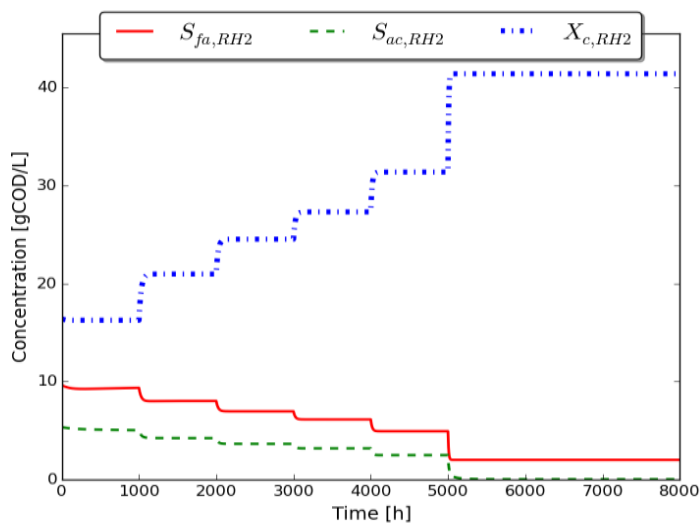

Fig. 14

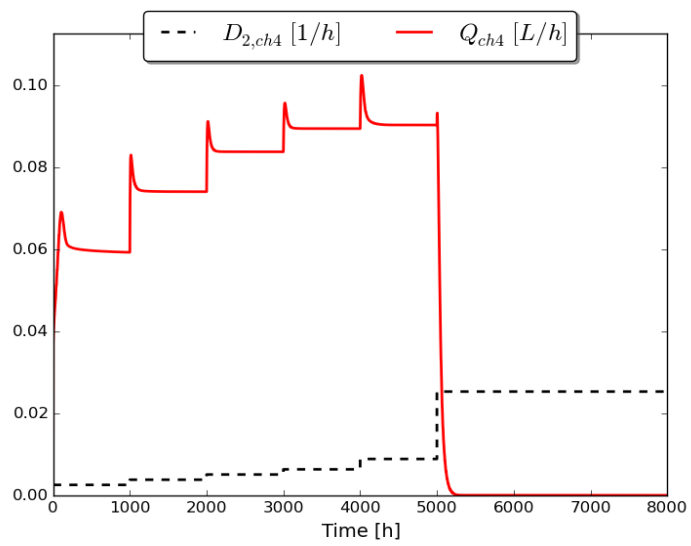

Fig. 13

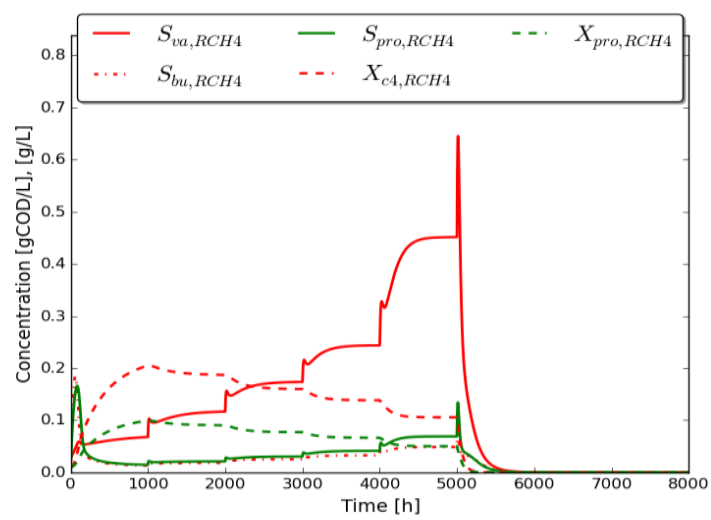

Fig. 15 


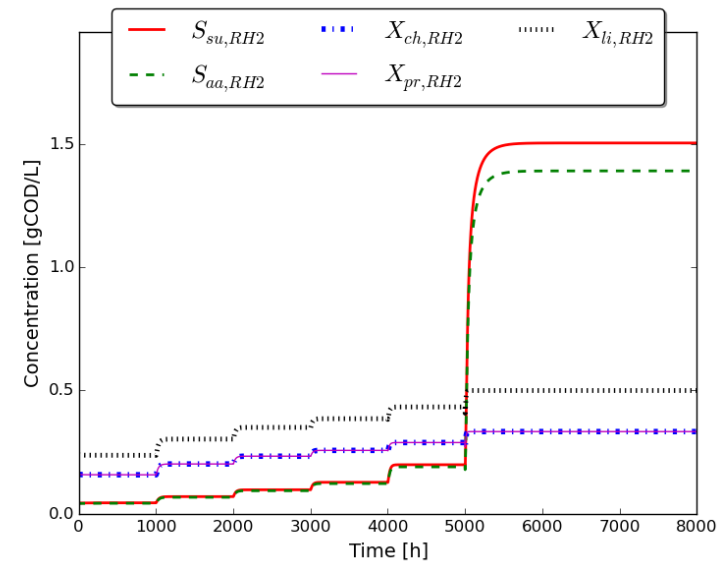

Fig. 16

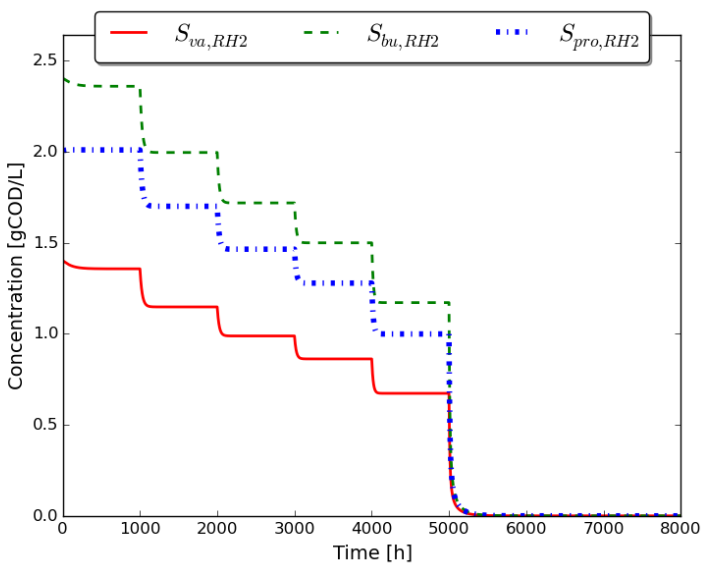

Fig. 18

\section{CONCLUSION}

In this paper a new mathematical model is proposed. This model describes the process of simultaneous production of hydrogen and methane by $\mathrm{AD}$ of organic wastes in a cascade of two CSTR bioreactors with different volumes. The proposed model is developed by reducing the universal but very complicated ADM1. The investigation of the inputoutput static characteristics $Q_{h 2}$ and $Q_{c h 4}$ shows existence of maxima with respect to hydrogen and methane. This fact is important for the practical applications and will be further used in optimising the bioreactors to achieve maximal production of either hydrogen and methane. The model also allows finding the optimal ratio between the volumes $\left(V_{1}\right.$ and $\left.V_{2}\right)$ of the two bioreactors subject to the same optimisation goal.

Currently two-phase AD of lignocellulosic waste (wheat straw) with hydrogen and methane production in laboratory conditions are in experimental studies using some theoretical results obtained with the above presented model.

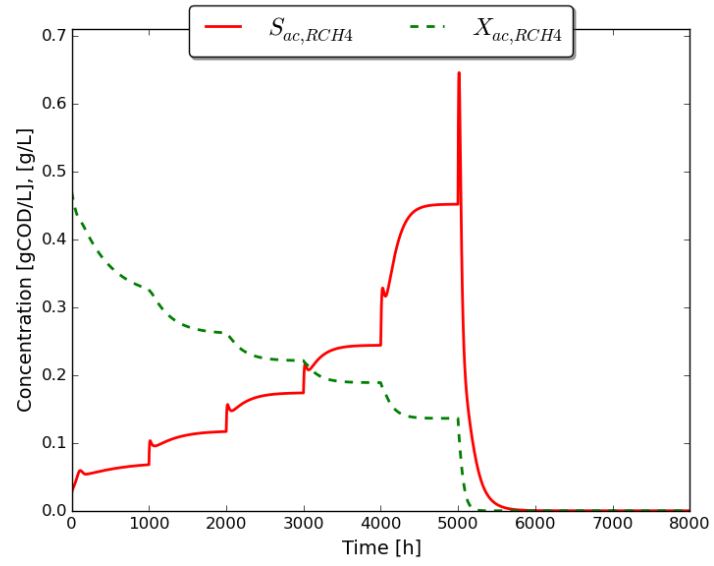

Fig. 17

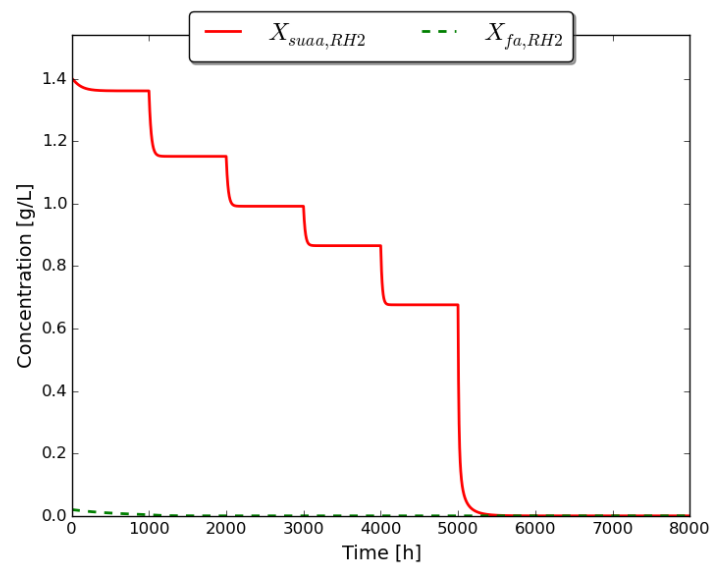

Fig. 19

ACKNOWLEDGMENTS: This research has been supported by the Bulgarian Science Fund under contract No. DFNI-E02/13

\section{REFERENCES}

1. Aceves-Lara, C.-A., Latrille, E., and Steyer, J.-P. Optimal control of hydrogen production in a continuous anaerobic fermentation bioreactor Int. J. of Hydrogen Energy, 35, 2010, I0710-I0718.

2. Ahring, B. (ed.) Biomethanation, I and II Springer-Verlag, Berlin-Heidelberg, 2003.

3. Ausiello, A. et al. Biohydrogen production by dark fermentation of Arundo donax using a new methodology for selection of $\mathrm{H}_{2}$-producing bacteria. Int. J. Hydrogen Energy, 42, 2017, 30599-30612.

4. Batstone, D. J., Keller, J., Angelidaki, I., Kalyuzhnyi, S. Pavlostathis, V., S. G., Rozzi, A., Sanders, W. T. Siegrist, M., H., and V. A. Vavilin. The IWA Anaerobic Digestion Model No. 1(ADM1). Water Sci \& Technology, 45 (10), 2002, 65-73.

5. Bernardo Ruggeri, Tonia Tommasi, Sara Sanfilippo, BioH2\&BioCH4 through anaerobic 
digestion (From research to full-scale applications), Springer, 2015, $215 \mathrm{p}$.

6. Blumensaat F., Keller J. Modelling of twostage anaerobic digestion using the IWA Anaerobic Digestion Model No 1 (ADM1), Water Research, 39, 2005, 171-183.

7. Chorukova E, Simeonov I, Mathematical modeling of the anaerobic digestion in two-stage system with production of hydrogen and methane including three intermediate products, Int. J. Hydrogen Energy, 2019, https://doi.org/10.1016/j.ijhydene.2019.01.2284. 229 SJR: 1.116

8. Chu, Chen-Yeon, Wang Zin-Fen. Dairy cow solid waste hydrolysis and hydrogen/methane productions by anaerobic digestion technology, Int. J. Hydrogen Energy, 42, 2017, 30591-98

9. Denchev, D., Simeonov, I., Hubenov, V., Kabaivanova, L. Biohydrogen production from lignocellulosic waste with anaerobic bacteria. Proc. ICWEE'2016, Burgas, Bulgaria, 01-03 June 2016, $7-12$

10. Deublein, D. and Steinhauser, A., 2008 Biogas from waste and renewable resources, Weinheim

11. Dochain, D., and Vanrolleghem, P., 2001 Dynamical modeling and estimation in wasterwater treatement process. IWA Publishing, London

12. Fenghao Cui, Seungho Lee, Moonil Kim, 2011, Removal of organics and nutrients from food wastewater using combined thermophilic two-phase anaerobic digestion and shortcut biological nitrogen removal, Water Research, 45, 5279-5286

13. Gerardi, M.H., 2003 The microbiology of anaerobic digesters. John Wiley\&Sons, Inc. New Jersey

14. Guo, X.M. et al., 2010, Hydrogen production from agricultural waste by dark fermentation: a review, Int. J. Hydrogen Energy, 36, 10660-73

15. H. Zhu, M. Béland, 2006, Evaluation of alternative methods of preparing hydrogen producing seeds from digested wastewater sludge. Int. J. Hydrogen Energy 31, 1980-1988

16. H.H.P. Fang, T. Zhang, H. Liu, 2002, Microbial diversity of a mesophilic hydrogen-producing sludge. Appl. Microbiol. Biotechnol. 58, 112-118

17. Hussy, F.R. Hawkes, R. Dinsdale, D.L. Hawkes, 2005, Continuous fermentative hydrogen production from sucrose and sugarbeet. Int. J. Hydrogen Energy 30, 471-483

18. J.J. Lay, K.S. Fan, J.1 Chang, C.H. Ku, 2003, Influence of chemical nature of organic wastes on their conversion to hydrogen by heat-shock digested sludge. Int. J. Hydrogen Energy 28(12), 1361-1367

19. Jayalakshmi, Kurian Joseph, V. Sukumaran, 2009, Bio hydrogen generation from kitchen waste in an inclined plug flow reactor, Int. J. Hydrogen Energy 34, 8854-8858

20. Lin, C.Y., C.H. Lay, 2005, A nutrient formulation for fermentative hydrogen production using anaeobic sewage sludge microflora. Int. J. Hydrogen Energy 30, 285-292

21. Borisov, M., Dimitrova, N., Simeonov, I., 2016, Mathematical Modelling of Anaerobic Digestion with Hydrogen and Methane Production, Preprints of the 6th IFAC Conference on Foundations of Systems Biology in Engineering, The International Federation of Automatic Control, Magdeburg, Germany, October 912, 2016, (TuPP.2: 1-8), IFAC-PapersOnLine, Vol. 49, Issue $26,231-238$

22. Mosier, N., C. Wyman, B. Dale, R. Elander, Y.Y. Lee, M. Holtzapple, M. Ladisch, 2005, Features of promising technologies for pretreatment of lignocellulosic biomass. Bioresour. Technol. 96(6), 673-686

23. Nasr, N. et al., 2013, Application of artificial neu-ral networks for modeling of biohydrogen production, Int. J. Hydrogen Energy, 38, 3189-3195

24. Pakarinen, O. M. et al., 2011, Hydrogen and methane yields of untreated, wter-extracted and acid $(\mathrm{HCl})$ treated maize in one- and two-stage batch assays. Int. J. Hydrogen Energy, 36, 14401-144407

25. Bakonyi, P., J. Dharmaraja, S. Shobana, L. Kook, T. Rozsenberszki, N. Nemestothy, R. Banu J, K. Belafi-Bako, G. Kumar, Leachate valorization in anaerobic biosystems: Towards the realization of waste-to-energy concept via biohydrogen, biogas and bioelectrochemical processes, Int. J. Hydrogen Energy, 2019

26. Oremland, R.S., Biology of Anaerobic Microorganisms, ed. by A.J.B. Zehnder. Biogeochemistry of Methanogenic Bacteria, Wiley Press, New York, 1988, 641-705

27. R.Y. Stanier, L. John Ingraham, L. Mark, P.D. Wheelis, P.R. Painter, The Microbial World, Prentice Hall, Englewood Cliffs, 1986.

28. Rafieenia R., A. Pivato, M. C. Lavagnolo, 2018, Effect of inoculum pre-treatment on mesopphilic hydrogen and methane production from food waste using two-stage anaerobic digestion, Int. J. Hydrogen Energy, 43, 12013-12022

29. Rajagopal, R., M. Rahman Choudhury, N. Anwar, B. Goyette and Md. Saifur Rahaman, Influence of pre-hydrolysis on sewage treatment in an up-flow anaerobic sludge BLANKET (UASB) reactor: A Review, Waste, 2019, 11, 372; doi:10.3390/w11020372

30. Rosen, Ch., and Jeppsson, U., Aspects on ADM1 implementation within the BSM2 framework. CODEN: LUTEDX, TEIE-7224, 2006, 1-35.

31. Mirzoyan, S., A. Trchounian, K. Trchounian, 2019, Hydrogen production by 
Escherichia coli during anaerobic utilization of mixture of lactose and glycerol: Enhanced rate and yield, prolonged, production, Int. J. Hydrogen Energy

32. Schievano, A., Tenca, A., Lonati, S., Manzini, E., Adani, F., 2014, Can two-stage instead one-stage anaerobic digestion really increase energy recovery from biomass? Applied Energy, 124, 335-342

33. S. G. Shin, G. Han, J. Lim, Ch. Lee, S. Hwang, 2010, A comprehensive microbial insight into two-stage anaerobic digestion of food waste-recycling wastewater, Water Research, 44, 4838-4849

34. Simeonov I., 2005, Laboratory studies and mathematical modeling of the anaerobic digestion in a cascade of two bioreactors, Ecological Engineering and Environment Protection, 2, 51-57 (in Bulgarian)

35. Simeonov I., E. Chorukova, Mathematical Modeling of the Anaerobic Digestion with Production of Hydrogen and Methane, The 4th International Conference on Water, Energy and Environment (ICWEE), Burgas,| June 1-2, 2016, 32-38

36. Simeonov I., S. Stoyanov, Modelling and extremum seeking control of a cascade of two anaerobic bioreactors, Proc. of the $16^{\text {th }}$ Int. Conf. on Control Systems and Computer Science, Bucharest, 22-25 May 2007, on CD

37. Simeonov, I., 1999, Mathematical modeling and parame-ters estimation of anaerobic fermentation process. Bio-process Engineering, 21 (4), 377-381

38. Simeonov, I., 2010, Ch., 2. Dynamical modeling and estimation in wasterwater treatement

\section{Milen Borisov}

Institute of Mathematics and Informatics

Bulgarian Academy of Sciences

B1. 8, Georgi Bonchev Str.

Sofia, 1113, Bulgaria

\section{Address for correspondence:}

Ivan Simeonov

e-mail: issim@microbio.bas.bg process. In St. Tzonkov (ed.), Contemporary Approaches to Mod-elling, Optimisation and Control of Biotechnological Processes, Prof. M. Drinov Acad. Publ. House, Sofia

39. Simeonov, I., Noykova, N., and Gyllenberg, M., 2007, Identification and extremum seeking control of the anaerobic digestion of organic wastes. Cybernetics and information technologies, 7 (2), 73-84

40. Brock, T.D., M.T. Madigan, J.M. Martino, J. Parker, Biology of microrganisms, Prentice-Hall, New york, 1994

41. Achinasb, V., G. Jan Willem, 2017, A Technological Overview of Biogas Production from Biowaste, Engineering 3, 299-30722

42. Wang, H.-H., Krstic, M., and Bastin, G., Optimizing bioreactors by extremum seeking. Int. J. Adapt. Control Signal Process, 13, 1999, 651-669.

43. Wang, J., and Wan, W., Kinetic models for fermentative hydrogen production: A review. Int. J. Hydrogen Energy, 34, 2009, 3313-3323

44. Wen-Wei Li, Han-Qing Yu, Advances in Energy-Producing Anaerobic Biotechnologies for Municipal Wastewater Treatment, Engineering 2, 2016, 438-446

45. Wiggins, S., 1990, Introduction to applied nonlinear dynamical systems and chaos. Springer Verlag, New York

46. Xing Wang, You-cai Zhao, A bench scale study of fermentative hydrogen and methaneproduction from food waste in integrated two-stage process, Int. J. Hydrogen Energy 34, 2009, 245-254

\section{Dencho Denchev, Ivan Simeonov}

The Stephan Angeloff Institute of Microbiology

Bulgarian Academy of Sciences

B1. 26, G. Bonchev Str.

Sofia, 1113, Bulgaria 